https://helda.helsinki.fi

\title{
Quasiconformal maps with controlled Laplacian
}

\section{Kalaj, David}

2019-03

Kalaj , D \& Saksman , E 2019 , ' Quasiconformal maps with controlled Laplacian ' , Journal d'Analyse Mathematique , vol. 137 , no. 1 , pp. 251-268 . https://doi.org/10.1007/s11854-018-0072-5

http://hdl.handle.net/10138/312568

https://doi.org/10.1007/s11854-018-0072-5

cc_by

acceptedVersion

Downloaded from Helda, University of Helsinki institutional repository.

This is an electronic reprint of the original article.

This reprint may differ from the original in pagination and typographic detail.

Please cite the original version. 


\title{
QUASICONFORMAL MAPS WITH CONTROLLED LAPLACIAN
}

\author{
DAVID KALAJ AND EERO SAKSMAN
}

\begin{abstract}
Aвstract. We establish that every $K$-quasiconformal mapping $w$ of the unit disk $\mathbb{D}$ onto a $C^{2}$-Jordan domain $\Omega$ is Lipschitz provided that $\Delta w \in$ $L^{p}(\mathbb{D})$ for some $p>2$. We also prove that if in this situation $K \rightarrow 1$ with $\|\Delta w\|_{L^{p}(\mathbb{D})} \rightarrow 0$, and $\Omega \rightarrow \mathbb{D}$ in $C^{1, \alpha}$-sense with $\alpha>1 / 2$, then the bound for the Lipschitz constant tends to 1 . In addition, we provide a quasiconformal analogue of the Smirnov theorem on absolute continuity over the boundary.
\end{abstract}

\section{INTRODUCTION}

Recall that the map $w: \mathbb{D} \rightarrow \mathbb{C}$ of the unit disc to the complex plane is quasiconformal if it is a sense preserving homeomorphism that has locally $L^{2}$-integrable weak partial derivatives, and it satisfies for almost every $z \in \mathbb{D}$ the distortion inequality $\left|w_{\bar{z}}\right| \leq k\left|w_{z}\right|$, where $k<1$. In this situation we say that $w$ is $K$-quasiconformal, with $K:=(1+k) /(1-k)$. We refer to [2] and [4] for basic notions and results of the quasiconformal theory. Quasiconformal self-maps of the disc, even when locally $C^{2}$-smooth inside $\mathbb{D}$, need not to be Lipschitz. However, in the situation where $w: \mathbb{D} \rightarrow \mathbb{D}$ is a quasiconformal homeomorphism that is also harmonic, Pavlović [18] proved that $f$ is biLipschitz. Many generalisations of this result for harmonic maps heve been proven since, we refer e.g. to [13] and [5] and the references therein.

Our paper addresses the following problem: how much one can relax the condition of harmonicity of the quasiconformal map $w$, while still being able to deduce the Lipschitz property of $w$-in this situation it is less natural to inquire $w$ to be bi-Lipschitz. Better insight to this kind of questions ought to be useful also in applications to non-linear elasticity. A natural measure for the deviation from harmonic functions is to consider $\|\Delta w\|_{L^{p}(\mathbb{D})}$ for some $p \geq 1$ and ask whether finiteness of this quantity enables one to make the desired conclusion. Our first result yields the following:

Theorem 1. Assume that $g \in L^{p}(\mathbb{D})$ and $p>2$. If $w$ is a $K$-quasiconformal solution of $\Delta w=g$, that maps the unit disk onto a bounded Jordan domain

Date: November 02, 2014.

E.S. was supported by the Finnish CoE in Analysis and Dynamics Research, and by the Academy of Finland, projects $113826 \& 118765$. 
$\Omega \subset \mathbb{C}$ with $C^{2}$-boundary, then $w$ is Lipchitz continuous. The result is sharp since it fails in general if $p=2$.

The proof is given in Section 2 ,

Our second result shows that, in the setting of Theorem 1, the Lipschitz constant of a normalised map $f$ becomes arbitrarily close to 1 if the image domain $\Omega$ approaches the unit disc in a suitably defined $C^{1, \alpha}$-sense, and if deviations both from conformality and harmonicity tend to zero. Below we identify $[0,2 \pi)$ and the boundary of the unit disc $\mathbb{T}$ in the usual way.

Theorem 2. Let $p>2$ and assume that $w_{n}: \mathbb{D} \rightarrow \Omega_{n}$ is a $K_{n}$-quasiconformal normalised map normalised by $w(0)=0$, and with

$$
\lim _{n \rightarrow \infty} K_{n}=1 \quad \text { and } \quad \lim _{n \rightarrow \infty}\left\|\Delta w_{n}\right\|_{L^{p}(\mathbb{D})}=0 .
$$

Moreover, assume that for each $n \geq 1$ the bounded Jordan domain $\Omega_{n}$ approaches the unit disc in the $C^{1, \alpha}$-bounded sense. More precisely, this means that there is a parametrisation

$$
\partial \Omega_{n}=\left\{f_{n}(\theta) \mid \theta \in \mathbf{T}\right\},
$$

where $f_{n}$ satisfies for some $\alpha>1 / 2$

$$
\left\|f_{n}(\theta)-e^{i \theta}\right\|_{L^{\infty}(\mathbf{T})} \rightarrow 0 \quad \text { as } \quad n \rightarrow \infty \quad \text { and } \quad \sup _{n \geq 1}\left\|f_{n}(\theta)\right\|_{C^{1, \alpha}(\mathbb{T})}<\infty .
$$

Then for large enough $n$ the function $w_{n}$ is Lipschitz, and moreover its Lipschitz constant tends to 1 as $n \rightarrow \infty$ :

$$
\lim _{n \rightarrow \infty}\|\nabla w\|_{L^{\infty}(\mathbb{D})}=1 .
$$

This result will be obtained as a corollary of slightly more general results in Section 3 below. Together, our Theorems 1 and 2 considerably improve the main result of the first author and Pavlović from [15], where it was instead assumed that $\Delta w \in C(\overline{\mathbb{D}})$. Other related results are contained in [14], we refer to [6] and references therein for other type of connections between quasiconformal and Lipschitz maps.

In order to state our last theorem, we recall the result of V. I. Smirnov, stating that a conformal mapping of the unit disk $\mathbf{U}$ onto a Jordan domain $\Omega$ with rectifiable boundary has a absolutely continuous extension to the boundary. This implies in particular that if $E \subset \mathbf{T}$ is a set of zero 1-dimensional Hausdorff measure then its image $f(E)$ is a set of zero 1dimensional Hausdorff measure in $\partial \Omega$. Further, this result has been generalized for the class of q.c. harmonic mapping by several authors (see e.g. [17, 11]). On the other hand if we assume that $f$ is merely quasiconformal, then its boundary function need not be in general an absolutely continuous function. In Section 4 we prove the following generalization of Smirnov's 
theorem for quasiconformal mappings, subject again to an size condition on their Laplacian:

Theorem 3. Let $f$ be a quasiconformal mapping of the unit disk onto a Jordan domain with rectifiable boundary. Assume that $\Delta f$ is locally integrable and satisfies

$$
|\Delta f(z)| \leq C(1-|z|)^{-a}
$$

for some constants $a<1$, and $C<\infty$. Then $f_{\mid \mathrm{T}}$ is an absolutely continuous function. The result is optimal: there is a quasiconformal self-map $f$ : $\mathbb{D} \rightarrow \mathbb{D}$, with non-absolutely continuous boundary values, and such that $f \in C^{\infty}(\mathbb{D})$ and with $|\Delta f(z)| \leq C(1-|z|)^{-1}$ in $\mathbb{D}$.

Another variant of the proof of the previous theorem goes as follows:

Corollary 1. If $f$ is a quasiconformal mapping of the unit disk onto a Jordan domain with rectifiable boundary such that $\Delta f \in L^{p}(\mathbb{D})$ for some $p>1$, then $f_{\mid \mathbf{T}}$ is an absolutely continuous function. The claim fails in general if $p<1$.

Further comments, generalizations and open questions related to the above results are included in in Sections $2,4$.

2. Proof of Theorem 1) Lipschitz-Property of QC-SOlutions to $\Delta f=g$

In what follows, we say that a bounded Jordan domain $\Omega \subset \mathbb{C}$ has $C^{2}$ boundary if it is the image of the unit disc $\mathbb{D}$ under a $C^{2}$-diffeomorphism of the whole complex plane onto itself. For planar Jordan domains this is well-known to be equivalent to the more standard definition, that requires the boundary to be locally isometric to the graph of a $C^{2}$-function on $\mathbb{R}$. In what follows, $\Delta$ refers to the distributional Laplacian. We shall make use of the following well-known fact, whose proof we recall:

Lemma 1. Assume that $w \in C(\overline{\mathbb{D}})$ is such that $\|\Delta w\|_{L^{p}(\mathbb{D})}<\infty$ with $p>1$.

(i) In case $p>2$ one has $\|\nabla w\|_{L^{\infty}(B(0, r))}<\infty$ for any $r<1$. Moreover, if $w_{\mid \partial \mathbb{D}}=0$, then there is $C_{p}<\infty$ so that

$$
\|\nabla w\|_{L^{\infty}(\mathbb{D})} \leq C_{p}\|\Delta w\|_{L^{p}(\mathbb{D})} .
$$

(ii) If $w_{\mid \partial \mathbb{D}}=0$, and $1<p<2$, then $\|\nabla w\|_{2 p /(2-p)}<\infty$.

Proof. By the classical representation we have for $|z|<1$

$$
w(z)=\frac{1}{2 \pi} \int_{0}^{2 \pi} P\left(z, e^{i \varphi}\right) w\left(e^{i \varphi}\right) d \varphi+\int_{\mathbf{U}} G(z, \omega) \Delta w(\omega) d A(\omega),
$$


where $P$ stands for the Poisson kernel and $G(z, \omega):=\frac{1}{2 \pi} \log \left|\frac{1-z \bar{\omega}}{z-\omega}\right|$ for the Green's function of $\mathbb{D}$. We observe first that since $G$ is real-valued, $|\nabla G|=$ $2\left|\partial_{z} G\right|$ so that

$$
|\nabla G(z, \omega)|=\frac{1}{2 \pi}\left|\frac{-\bar{\omega}}{1-z \bar{\omega}}-\frac{1}{z-\omega}\right| \leq \frac{1}{\pi|z-\omega|} .
$$

Hence an application of Hölder's inequality shows that the second term in (2) has uniformly bounded gradient in $\mathbb{D}$. To conclude part (i) it suffices to to observe that the first term vanishes if $w_{\mid \partial \mathbb{D}}=0$, and in the general case case it has uniformly bounded gradient in compact subsets of $\mathbb{D}$. Finally, part (ii) follows immediately from (3) by the standard mapping properties of the Riesz potential $I_{1}$ with the kernel $|z-\omega|^{-1}$, see [21].

Proof of Theorem 1. It would be natural to try to generalise the ideas in [14] where differential inequalities were applied while treating related problems. However, it turns out that the approach of [12], where the use of distance functions was initiated, is flexible enough for further development.

In the sequel we say $a \approx b$ if there is a constant $C \geq 1$ such that $a / C \leq$ $b \leq C a$; and we say $a \lesssim b$ if there is a constant $C>0$ such that $a \leq C b$.

By our assumption on the domain, we may fix a diffeomorphism $\psi: \bar{\Omega} \rightarrow$ $\overline{\mathbb{D}}$ that is $C^{2}$ up to the boundary. Denote $H:=1-|\psi|^{2}$, whence $H$ is $C^{2}$ smooth in $\bar{\Omega}$ and vanishes on $\partial \Omega$ with $|\nabla H| \approx 1$ in a neighborhood of $\partial \Omega$. We may then define $h: \mathbb{D} \rightarrow[0,1]$ by setting

$$
h(z):=H \circ w(z)=1-|\psi(w(z))|^{2} \text { for } z \in \mathbb{D} .
$$

The quasiconformality of $f$ and the behavior of $\nabla H$ near $\partial \Omega$ imply that there is $r_{0} \in(0,1)$ so that the weak gradients satisfy

$$
|\nabla h(x)| \approx|\nabla w(x)| \text { for } r_{0} \leq|x|<1 .
$$

Moreover, by Lemma11(i) we have $|\nabla h(x)| \lesssim|\nabla w(x)| \leq C$ for $|x| \leq r_{0}$. It follows that for any $q \in(1, \infty]$ we have that

$$
\nabla h \in L^{q}(\mathbb{D}) \quad \text { if and only if } \nabla w \in L^{q}(\mathbb{D}) .
$$

A direct computation, simplified by the fact that $H$ is real valued, yields that

(6)

$\Delta h=\Delta(H \circ w))=(\Delta H)(w)\left(\left|w_{z}\right|^{2}+\left|w_{\bar{w}}\right|^{2}\right)+2 \operatorname{Re}\left(4 H_{z z}(w) w_{z} w_{\bar{z}}+H_{z}(w) \Delta w\right)$.

Especially, since $H \in C^{2}(\bar{D})$ we have

$$
|\Delta h| \lesssim|\nabla w|^{2}+|g|
$$

The higher integrability of quasiconformal self-maps of $\mathbb{D}$ makes sure that $\nabla(\psi \circ w) \in L^{q}(\mathbb{D})$ for some $q>2$, which implies that $\nabla w \in L^{q}(\mathbb{D})$. By combining this with the fact that $g \in L^{p}(\mathbb{D})$ with $p>2$, we deduce that 
$\Delta h \in L^{r}(\mathbb{D})$ with $r=\min (p, q / 2)>1$. This information is not enough to us in case $q \leq 4$, but we will actually show that one may improve the situation to $q>4$ via a bootstrapping argument based on the following observation: in our situation

(8) if $\nabla w \in L^{q}(\mathbb{D})$ with $2<q<4$, then actually $\nabla w \in L^{2 q /(4-q)}(\mathbb{D})$.

In order to prove (8), assume that $\nabla w \in L^{q}(\mathbb{D})$ for an exponent $q \in(2,4)$. Then (7) and our assumption on $g$ verify that $\Delta h \in L^{q / 2}(\mathbb{D})$. Since $h$ vanishes continuously on the boundary $\partial \mathbb{D}$, we may apply Lemma11(ii) to obtain that $\nabla h \in L^{2 q /(4-q)}(\mathbb{D})$ which yields the claim according to (5).

We then claim that in our situation one has $\nabla w \in L^{q}(\mathbb{D})$ with some exponent $q>4$. For that end, fix an exponent $q_{0}>2$ obtained from the higher integrability of the quasiconformal map $w$ so that $\nabla w \in L^{q_{0}}(\mathbb{D})$. By diminishing $q_{0}$ if needed, we may well assume that $q_{0} \in(2,4)$ and $q_{0} \notin$ $\left\{2^{n} /\left(2^{n-1}-1\right), n=3,4, \ldots\right\}$. Then we may iterate (8) and deduce inductively that $\nabla w \in L^{q_{k}}(\mathbb{D})$ for $k=0,1,2 \ldots k_{0}$, where the indexes $q_{k}$ satisfy the recursion $q_{k+1}=\frac{2 q_{k}}{4-q_{k}}$ and $k_{0}$ is the first index such that $q_{k_{0}}>4$. Such an index exists since by induction we have the relation $\left(1-2 / q_{k+1}\right)=2^{k}\left(1-2 / q_{0}\right)$, for $k \geq 0$.

Thus we may assume that $\nabla w \in L^{q}(\mathbb{D})$ with $q>4$. At this stage (7) shows that $\Delta h \in L^{p \wedge(q / 2)}(\mathbb{D})$. As $p \wedge(q / 2)>2$, Lemma11(ii) verifies that $\nabla h$ is bounded. Finally, by (5) we have the same conclusion for $\nabla w$, and hence $w$ is Lipschitz as claimed.

In order to verify the sharpness of the result, consider the following map

$$
w_{0}(z)=z \log ^{a}\left(\frac{e}{|z|^{2}}\right),
$$

where $a \in(0,1 / 2)$ is fixed. Then $w_{0}$ is a self-homeomorphism of $\mathbb{D}$ that is quasiconformal with continuous Beltrami-coefficient since we may easily compute $\left(w_{0}\right)_{z}=\log ^{a-1}\left(\frac{e}{|z|^{2}}\right) \log \left(\frac{e^{1-a}}{|z|^{2}}\right)$ and $\left(w_{0}\right)_{\bar{z}}=-a \frac{z}{\bar{z}} \log ^{a-1}\left(\frac{e}{|z|^{2}}\right)$ so that the complex dilatation of $w_{0}$ satisfies

$$
\left|\mu_{w_{0}}(z)\right|=\left|-a \frac{z}{\bar{z}}\left(\log \left(\frac{e^{1-a}}{|z|^{2}}\right)\right)^{-1}\right| \leq \frac{a}{1-a}<1 .
$$

In addition, we see that $\Delta w_{0} \in L^{2}(\mathbb{D})$ since

$$
\begin{aligned}
\left|\Delta w_{0}(z)\right| & =\left|4 \frac{d}{d \bar{z}}\left(w_{0}\right)_{z}(z)\right|=\left|\frac{4 a}{\bar{z}} \log ^{a-2}\left(\frac{e}{|z|^{2}}\right)\left((a-1)-\log \left(\frac{e}{|z|^{2}}\right)\right)\right| \\
& \lesssim|z|^{-1}\left(\log \left(\frac{e}{|z|^{2}}\right)\right)^{a-1} .
\end{aligned}
$$

Finally, it remains to observe that $w$ is not Lipschitz at the origin. 
Remark 2.1. If one invokes the known sharp $L^{p}$-integrability results of qcmaps (due to Astala, see [4, Thm 13.2.3 ]) one sees that in the above proof no iteration is needed in case $K<2$. One should also observe that the counterexample given above in the case $p=2$ is based already on the behaviour of $w$ near origin, not to any boundary effect, so in this sense Theorem 1 is quite sharp. We have not seriously pursued the optimality question related to $C^{2}$-regularity assumption on $\Omega$. Here we simply observe that an easy modification of the proof along the lines of Section 3 yields slightly stronger result, where instead of $C^{2}$-condition one only assumes that $\partial \Omega$ is $C^{1, \alpha}$-smooth for any $\alpha \in(0,1)$.

Remark 2.2. Assume that $w: B(0,1) \rightarrow B(0,1)$ is quasiconformal where $B(0,1) \subset \mathbb{R}^{d}$ is the $d$-dimensional unit ball, $d \geq 3$ and such that $\Delta w_{k} \in$ $L^{p}(B(0,1))$ with $p>n$ for each component of $w$ (here $\left.k=1, \ldots, d\right)$. Then the above proof applies with some modifications and shows that $w$ is Lipschitz. Actually, in a recent preprint [5] Astala and Manojlović proved that quasiconformal harmonic gradient mapping of the unit ball $B^{3}$ on to itself are bi-Lipschitz. They also provide a short new proof of the Lipschitzproperty of quasiconformal harmonic maps of the unit ball onto a domain with $C^{2}$ boundary on $\mathbb{R}^{d}$ (c.f. [14, Theorem C]). The results of [5] and of the present paper were obtained independently.

\section{Proof of Theorem 2, quantitative bounds as $\Omega \rightarrow \mathbb{D}$}

We start with an auxiliary lemma.

Lemma 2. There exists a function $\psi:(1,2) \rightarrow \mathbb{R}^{+}$with the following property: If $w: \mathbb{D} \rightarrow \mathbb{D}$ is a $K$-quasiconformal self-map normalised with $\psi(0)=0$, then

$$
\left\|\left|w_{z}\right|^{2}+\left|w_{\bar{z}}\right|^{2}-1\right\|_{L^{3}(\mathbb{D})} \leq \psi(K) .
$$

Moteover, $\lim _{K \rightarrow 1^{+}} \psi(K)=0$.

Proof. By the sharp area distortion $\|\nabla w\|_{L^{6}(\mathbb{D})}<\infty$ for $K<3 / 2$. By reflecting $w$ over the boundary $\partial \mathbb{D}$ we may also assume that $w$ extends to a $K$-quasiconformal map (still denoted by $w$ ) to the whole plane. By rotation of needed, we may also impose the condition that $w(1)=1$. Furthermore, we may even assume that $w_{\mathbb{C} \backslash B\left(0, e^{3 \pi}\right)}$ is the identity map, since we may use standard quasiconformal surgery (choose $k=(K-1) /(K+1)$ and $\alpha=2 k$ in [4. Theorem 12.7.1]) to produce $\frac{3 K-1}{3-K}$-quasiconformal modification (still denoted by $w$ ) that equals to the original function $w$ in $\mathbb{D}$ and satisfies $w(z)=z$ for $|z| \geq e^{3 \pi}$. Especially, it is a principal solution. Since $\frac{3 K-1}{3-K} \rightarrow 1$ as $K \rightarrow 1$, and we are interested only on small values of $K$, it is thus enough to prove the corresponding claim for principal solutions with complex dilatation supported in $B\left(0, e^{3 \pi}\right)$. 
Denote by $M$ the norm of the Beurling operator on $L^{6}(\mathbb{C})$. Fix $R_{0}>0$ and consider a principal solution $w$ to the Beltrami equation $w_{\bar{z}}=\mu w_{z}$ with $|\mu| \leq k<1 / 2 M$. Then we have the standard Neumann-series representation

$$
w_{\bar{z}}=\mu+\mu T \mu+\mu T \mu T \mu+\ldots \quad \text { and } \quad w_{z}-1=T w_{\bar{z}} .
$$

We thus obtain that

$$
\left\|w_{\bar{z}}\right\|_{L^{6}(\mathbb{C})} \leq\|\mu\|_{L^{6}(\mathbb{C})}\left(1+\frac{M}{2 M}+\left(\frac{M}{2 M}\right)^{2}+\ldots\right) \leq 2\|\mu\|_{L^{6}(\mathbb{C})} \leq C k^{1 / 6}
$$

and, a fortiori $\left\|w_{z}-1\right\|_{L^{6}(\mathbb{C})} \leq M C k^{1 / 6}=C^{\prime} k^{1 / 6}$. We obtain the desired $L^{3}$-estimate for $\left|f_{\bar{z}}\right|^{2}$ since $k \rightarrow 0$ as $K \rightarrow 1$. The estimate for $\left|f_{z}\right|^{2}-1$ follows by noting that $\left.|| f_{z}\right|^{2}-1|\leq| f_{z}-1 \mid\left(\left|f_{z}-1\right|+2\right)$ and applying Hölder's inequality.

Before proving the more general convergence result stated in the introduction it is useful to consider first the case where the image domain is fixed, and in fact equals $\mathbb{D}$.

Proposition 3.1. Assume that $p>2$. There exist a function

$$
[1, \infty) \times[0, \infty) \ni(K, t) \rightarrow \widetilde{C}_{p}(K, t)
$$

with the property: if $w: \mathbb{D} \rightarrow \mathbb{D}$ is a $K$-quasiconformal self map of the unit disc, normalised by $w(0)=0$, and with $\Delta w \in L^{p}(\mathbb{D})$, then one has

$$
\|\nabla w\|_{L^{\infty}(\mathbb{D})} \leq \widetilde{C}_{p}\left(K,\|\Delta w\|_{p}\right) .
$$

Moreover, the function $\widetilde{C}_{p}$ satisfies

$$
\lim _{K \rightarrow 1^{+}, t \rightarrow 0^{+}} \widetilde{C}_{p}(K, t)=1 .
$$

Proof. We follow the line to the proof of Theorem 1, in particular we employ its notation, but this time we strive to make the conclusion quantitative. We may well assume that $p \leq 3$. Let us then assume that $w$ is as in the assumption of the Proposition with $K<1+1 / 100$, say. In addition, we may freely assume that $w(1)=1$. As the image domain is $\mathbb{D}$, the function $h$ from the proof of Theorem 1, takes the form

$$
h(z)=1-|w(z)|^{2} .
$$

Let us write $h_{0}(z)=1-|z|^{2}$, which corresponds to $h$ when $w$ is the identity map. An application of (6) and Lemma 2 allow us to estimate

$$
\begin{aligned}
\left\|\Delta\left(h-h_{0}\right)\right\|_{L^{p}(\mathbb{D})} & =\left\|4\left(1-\left|w_{z}\right|^{2}\right)-4\left|w_{\bar{z}}\right|^{2}+2 \operatorname{Re}(\bar{w} g)\right\|_{L^{p}(\mathbb{D})} \\
& \leq 4\left\|\left(\left|w_{z}\right|^{2}-1\right)+\left|w_{\bar{z}}\right|^{2}\right\|_{L^{3}(\mathbb{D})}+\|g\|_{L^{p}(\mathbb{D})} \\
& \leq 4 \psi(K)+\|g\|_{L^{p}(\mathbb{D}) .}
\end{aligned}
$$


Lemma 1 implies that

$$
\left\|\nabla h-\nabla h_{0}\right\| \leq c_{p}\left(\psi(K)+\|g\|_{L^{p}(\mathbb{D})}\right) .
$$

The quasiconformality of $w$ implies that we have for almost every $z$ the estimate $|\nabla h(z)| \geq K^{-1}\left|\left(\nabla h_{0}\right)(w(z))\right||\nabla w(z)|$. Since $\left|\nabla h_{0}(z)\right|=2|z|$, we obtain by considering the annulus $1-\varepsilon \leq|z|<1$ with arbitrarily small $\varepsilon>0$ that

$$
\begin{aligned}
\limsup _{|z| \rightarrow 1^{-}}|\nabla w(z)| & \leq \frac{K}{2} \limsup _{|z| \rightarrow 1^{-}}\left(\left|\nabla h-\nabla h_{0}\right|+\left|\nabla h_{0}\right|\right) \\
& \leq \frac{c_{p} K}{2}\left(\psi(K)+\|g\|_{L^{p}(\mathbb{D})}\right)+K .
\end{aligned}
$$

We now write $w$ in terms of the standard Poisson decomposition $w=u+$ $f$, where $u$ is harmonic with $u_{\mid \partial \mathbb{D}}=w_{\mid \partial \mathbb{D}}$, the term $f$ has vanishing boundary values and it satisfies $\Delta f=\Delta w=g$ in $\mathbb{D}$. Then maximum principle applies to the subharmonic function $|\nabla u|=\left|u_{z}\right|+\left|u_{\bar{z}}\right|=\left|a^{\prime}\right|+\left|b^{\prime}\right|$, where $a$ and $b$ are analytic functions such that $u=a+\bar{b}$, together with Lemma 1 shows that $|\nabla w|$ is bounded by $c\|g\|_{L^{p}(\mathbb{D})}$. All, in all combing these observations with (11) we deduce that

$$
\begin{aligned}
\sup _{|z|<1}|\nabla w(z)| & \leq \limsup _{|z| \rightarrow 1^{-}}|\nabla u|+\sup _{|z|<1}|\nabla f(z)| \\
& \leq \limsup _{|z| \rightarrow 1^{-}}|\nabla w|+2 \sup _{|z|<1}|\nabla f(z)| \\
& \leq \frac{c_{p} K}{2}\left(\psi(K)+\|g\|_{L^{p}(\mathbb{D})}\right)+K+2 c_{p}\|g\|_{L^{p}(\mathbb{D})} .
\end{aligned}
$$

We may thus choose for small enough $K$

$$
\widetilde{C}_{p}(K, t)=K+\frac{c_{p} K}{2} \psi(K)+\frac{c_{p}(K+4)}{2} t,
$$

and the obtained bound has the desired behavior as $K \rightarrow 1$ and $t \rightarrow 0$.

Below Id stands for the identity matrix Id :=( $\left.\begin{array}{ll}1 & 0 \\ 0 & 1\end{array}\right)$. We refer to [21] for the standard definition of Sobolev spaces $W^{2, p}$ and for the Hölder(Zygmund)classes $C^{\alpha}$ and $C^{1, \alpha}$.

Definition 3.1. Let $p>2$. We say that the sequence of bounded Jordan domains $\Omega_{n} \subset \mathbb{C}$, with $0 \in \Omega_{n}$ for each $n \geq 1$, converges in $W^{2, p}$-controlled sense to the unit disc $\mathbb{D}$ if there exist sense-preserving diffeomorphisms $\Psi_{n}$ : $\mathbb{D} \rightarrow \Omega_{n}$, normalized by $\Psi_{n}(0)=0$, such that

(13) $\lim _{n \rightarrow \infty}\left\|D \Psi_{n}-\operatorname{Id}\right\|_{L^{\infty}(\mathbb{D})}=0, \quad$ and $\quad\left\|\Psi_{n}\right\|_{W^{2, p}(\mathbb{D})} \leq M_{0} \quad$ for all $n \geq 1$, where $M_{0}<\infty$, and

$$
\left\|\Delta \Psi_{n}\right\|_{L^{p}(\mathbb{D})} \rightarrow 0 \quad \text { as } n \rightarrow \infty .
$$


One should observe that since $\Psi_{n} \in W^{2, p}(D)$ with $p>2$ in the above definition, it follows automatically that $\nabla \Psi_{n} \in C(\bar{\Omega})$. Hence asking $\Psi_{n}$ to be a diffeomorphism makes perfect sense in terms and, in particular, by (13) the map $\Psi_{n}$ is a bi-Lipschitz for large enough $n$. Also, each $\Omega_{n}$ is a bounded $C^{1}$ - Jordan domain in the plane. It turns out that the above condition is in a sense symmetric with respect to the domains $\mathbb{D}$ and $\Omega$ :

Lemma 3. Assume that $\Omega_{n}$ tends to $\mathbb{D}$ in a controlled sense and $\left(\Psi_{n}\right)$ is the associated sequence of diffeomorphisms satisfying the conditions of definition 3.1 Then the inverse maps $\Phi_{n}:=\Psi_{n}^{-1}: \Omega_{n} \rightarrow \mathbb{D}$ satisfy

(15) $\lim _{n \rightarrow \infty}\left\|D \Phi_{n}-\mathrm{Id}\right\|_{L^{\infty}\left(\Omega_{n}\right)}=0$, and $\left\|\Phi_{n}\right\|_{W^{2, p}\left(\Omega_{n}\right)} \leq M_{0}^{\prime}$ for all $n \geq 1$,

together with

$$
\left\|\Delta \Phi_{n}\right\|_{L^{p}\left(\Omega_{n}\right)} \rightarrow 0 \quad \text { as } n \rightarrow \infty .
$$

Proof. Conditions (15) follows easily by employing the formulas for the derivatives of the implicit function, after first approximating by smooth functions. Note, in regards to condition (16), we note that in general the inverse of a harmonic diffeomorphism needs not to be harmonic, so (16) is not a direct consequence of (14). However, the first condition in (13) tells us that the maximal complex dilatation $k_{n}$ of $\Psi_{n}$ tends to 0 as $n \rightarrow \infty$, so that $\Psi_{n}$ is asymptotically conformal and this makes (16) more plausible. Indeed, a direct computations shows that for $C^{2}$-diffeo $\Psi: \mathbb{D} \rightarrow \Omega$ with maximal dilatation $k$ and controlled derivative $|D \psi|,\left|(D \psi)^{-1}\right| \leq C$, it holds that

$$
\Delta \Phi=A \circ \Phi,
$$

where (recall that the Jacobian can be expressed as $J_{\Psi}=\left|\Psi_{z}\right|^{2}-\left|\Psi_{\bar{z}}\right|^{2}$ )

$$
\begin{aligned}
A= & \frac{4}{\left(J_{\Psi}\right)^{3}}\left[-\Psi_{\bar{z}}\left(\overline{\Psi_{z \bar{z}}} J_{\Psi}-\overline{\Psi_{z}}\left(\overline{\Psi_{z}} \Psi_{z z}+\Psi_{z} \overline{\Psi_{z \bar{z}}}-\overline{\Psi_{\bar{z}}} \Psi_{z \bar{z}}-\Psi_{\bar{z}} \overline{\Psi_{\bar{z}}}\right)\right)\right. \\
& \left.+\Psi_{z}\left(\overline{\Psi_{z z}} J_{\Psi}-\overline{\Psi_{z}}\left(\overline{\Psi_{z}} \Psi_{z \bar{z}}+\Psi_{z} \overline{\Psi_{z z}}-\overline{\Psi_{\bar{z}}} \Psi_{\overline{z z}}-\Psi_{z} \overline{\Psi_{z \bar{z}}}\right)\right)\right]
\end{aligned}
$$

This formula is obtained by using as a starting point the identity $\Delta \Phi=$ $4(d / d \bar{z}) \Phi_{z}$ and expressing $\Phi_{z}$ in a standard manner in terms of the derivatives of $\Psi$. We next recall that $\Psi_{z}$ is bounded and $\left|\Psi_{\bar{z}}\right| \leq k \Psi_{z}$, and observe that in the right hand side of (17) the terms that do not directly contain either $\Psi_{z \bar{z}}$ or $\Psi_{\bar{z}}$ as a factor sum up to

$$
\overline{\Psi_{z z}}\left(J_{\Psi}-\left|\Psi_{z}\right|^{2}\right)=-\overline{\Psi_{z z}}\left|\Psi_{\bar{z}}\right|^{2},
$$

We obtain that

$$
|A| \lesssim k\left|D^{2} \Psi\right|+|\Delta \Psi|,
$$

and (16) follows by applying this on $\Psi_{n}$. 
We may now generalize Proposition 3.1 to include variable image domains that converge to the unit disc in controlled sense.

Theorem 4. Let $p>2$ and assume that the planar Jordan domains $\Omega_{n}$ converge to $\mathbb{D}$ in $W^{2, p}$-controlled sense. Moreover, assume that $w_{n}: \mathbb{D} \rightarrow$ $\Omega_{n}$ is a $K_{n}$-quasiconformal normalised map normalised by $w(0)=0$, and with

$$
\lim _{n \rightarrow \infty} K_{n}=1 \quad \text { and } \quad \lim _{n \rightarrow \infty}\left\|\Delta w_{n}\right\|_{L^{p}(\mathbb{D})}=0 .
$$

Then for large enough $n$ the function $w_{n}$ is Lipschitz, and moreover its Lipschitz constant tends to 1 as $n \rightarrow \infty$ :

$$
\lim _{n \rightarrow \infty}\left\|\nabla w_{n}\right\|_{L^{\infty}(\mathbb{D})}=1 .
$$

Proof. Let $\Psi_{n}: \mathbb{D} \rightarrow \Omega_{n}$ be the maps as in definition 3.1. By renumbering, if needed, we may assume that that $\left|\Psi_{n}^{\prime}(z)-1\right|<1 / 2$ for all $n$ and $z \in \mathbb{D}$. Write $\Phi_{n}=\Psi_{n}^{-1}$ and define

$$
\widetilde{w}_{n}:=\Psi^{-1} \circ w_{n}=\Phi_{n} \circ w_{n}: \mathbb{D} \rightarrow \mathbb{D} .
$$

Then $\widetilde{w}_{n}$ is $\widetilde{K}_{n}$-quasiconformal, with $\widetilde{K}_{n} \rightarrow 1$ as $n \rightarrow \infty$ by the first condition in (15). Fix an index $q \in(2, p)$. By conditions (13), (15) and Proposition 3.1, in order to prove (18) we just need to verify that

$$
\lim _{n \rightarrow \infty}\left\|\Delta \widetilde{w}_{n}\right\|_{L^{q(\mathbb{D})}}=0
$$

A computation yields that

$$
\begin{aligned}
\Delta \widetilde{w}_{n}= & \left(\Delta \Phi_{n}\right)\left(w_{n}\right)\left(\left|\left(w_{n}\right)_{z}\right|^{2}+\left|\left(w_{n}\right)_{\bar{z}}\right|^{2}\right) \\
& +4\left(\left(\Phi_{n}\right)_{z z}(w)\left(w_{n}\right)_{z}\left(w_{n}\right)_{\bar{z}}+\left(\Phi_{n}\right)_{\overline{z z}}\left(w_{n}\right) \overline{\left(w_{n}\right)_{z}\left(w_{n}\right)_{\bar{z}}}\right) \\
& +\left(\left(\Phi_{n}\right)_{z}\left(w_{n}\right) \Delta w_{n}+\left(\Phi_{n}\right)_{\bar{z}}\left(w_{n}\right) \overline{\Delta w_{n}}\right) \\
=: & S_{1}+S_{2}+S_{3},
\end{aligned}
$$

say.

Since $\left|D \Phi_{n}\right|$ remains uniformly bounded and we know that $\left\|\Delta w_{n}\right\|_{L^{p}(\mathbb{D})} \rightarrow$ 0 , we see that $\left\|S_{3}\right\|_{L^{p}(\mathbb{D})}$ tends to zero as $n \rightarrow \infty$, whence the same is true for the $L^{q}$-norm. Set $\widetilde{q}:=\sqrt{q p}$ so that $q<\widetilde{q}<p$. Since $\widetilde{w}_{n}$ is a normalized $K_{n}$-quasiconformal self-map of the unit disc $\mathbb{D}$, and $K_{n} \rightarrow 1$, we may assume (again by discarding small values of $n$ and relabeling, if needed) by the higher integrability of quasiconformal maps that $\int_{\mathbb{D}}\left|\nabla w_{n}\right|^{2(\widetilde{q} / q)^{\prime}}<C$ and $\int_{\Omega}\left(J_{w_{n}^{-1}}\right)^{(p / \widetilde{q})^{\prime}} d A(z)<C$ for all $n$. Here e.g. $(\widetilde{q} / q)^{\prime}$ stands for the dual exponent. Denoting $k_{n}=\left(K_{n}-1\right) /\left(K_{n}+1\right)$ we thus obtain for any measurable 
function $F$ on $\Omega$

$$
\begin{aligned}
\left.\left.\int_{\mathbb{D}}\left|F \circ w_{n}\right|\left(w_{n}\right)_{z}\right|^{2}\right|^{q} d A(z) & \leq\left(\int_{\mathbb{D}}\left|F \circ w_{n}\right|^{\widetilde{q}} d A(z)\right)^{q / \widetilde{q}}\left(\int_{\mathbb{D}}\left|\nabla w_{n}\right|^{2 \widetilde{q} / q)^{\prime}} d A(z)\right)^{1 /(\widetilde{q} / q)^{\prime}} \\
& \lesssim\left(\int_{\mathbb{D}}\left|F \circ w_{n}\right|^{\widetilde{q}} d A(z)\right)^{q \widetilde{q}} \leq\left(\int_{\Omega}|F|^{\widetilde{q}} J_{w_{n}^{-1}} d A(z)\right)^{q / \widetilde{q}} \\
& \lesssim\left(\int_{\Omega}|F|^{p} d A(z)\right)^{q / p}\left(\int_{\Omega}\left(J_{w_{n}^{-1}}\right)^{(p / \widetilde{q})^{\prime}} d A(z)\right)^{q /\left(\widetilde{q}(p / \widetilde{q})^{\prime}\right)} \\
& \leq\left(\int_{\Omega}|F|^{p} d A(z)\right)^{q / p} .
\end{aligned}
$$

By employing this formula and Lemma 3 we obtain immediately that

$$
\left\|S_{1}\right\|_{L^{q}(\mathbb{D})} \lesssim\left\|\Delta \Phi_{n}\right\|_{L^{p}(\Omega)} \rightarrow 0 \quad \text { as } \quad n \rightarrow \infty .
$$

In a similar vain

$$
\left\|S_{2}\right\|_{L^{q}(\mathbb{D})} \lesssim k_{n} \rightarrow 0 \quad \text { as } \quad n \rightarrow \infty .
$$

This ends the proof of the theorem.

We next examine what kind of convergence of the boundaries $\partial \Omega_{n} \rightarrow$ $\partial \mathbb{D}$ imply $W^{2, p}$-controlled convergence of the domains itself. First of all, given $\psi_{n}: \mathbb{D} \rightarrow \Omega$ as in Definition 3.1 we have $\Psi_{n} \in W^{2, p}(\mathbb{D})$, so by the standard trace theorem for the Sobolev spaces the induced map on the boundary satisfies $\Psi_{n \mid \partial \mathbb{D}} \in B_{p, p}^{2-1 / p}(\mathbb{D})$. On the other hand, for $p>2$ we may pick $\alpha, \alpha^{\prime} \in(1 / 2,1)$ so that

$$
C^{1, \alpha^{\prime}}(\partial \mathbb{D}) \subset B_{p, p}^{2-1 / p}(\mathbb{D}) \subset C^{1, \alpha}(\partial \mathbb{D}),
$$

see [23]. Hence about the best one can hope is to have a theorem where the boundary converges in $C^{1, \alpha}$ for some $\alpha>1 / 2$. In fact, this can be realized:

Theorem 5. Let $\left(\Omega_{n}\right)$ be a a sequence of bounded Jordan domains in $\mathbb{C}$ such that there is the parametrisation

$$
\partial \Omega_{n}=\left\{f_{n}(\theta) \mid \theta \in(0,2 \pi)\right\} .
$$

for each $n$, where $f_{n}$ satisfies for some $\alpha>1 / 2$

$$
\left\|f_{n}(\theta)-e^{i \theta}\right\|_{L^{\infty}(\mathbf{T})} \rightarrow 0 \quad \text { as } \quad n \rightarrow \infty \quad \text { and } \quad \sup _{n \geq 1}\left\|f_{n}(\theta)\right\|_{C^{1, \alpha}(\mathbb{T})}<\infty .
$$

Then the sequence $\left(\Omega_{n}\right)$ converges to $\mathbb{D}$ in $W^{2, p}$-controlled manner. In particular, the conclusion of Theorem 4 holds true for the sequence $\left(\Omega_{n}\right)$.

Proof. Let us first observe that instead of (21) we may fix $\alpha^{\prime} \in(1 / 2, \alpha)$ and assume that

$$
\left\|f_{n}(\theta)-e^{i \theta}\right\|_{C^{1, \alpha^{\prime}}} \rightarrow 0 \quad \text { as } \quad n \rightarrow \infty
$$

Namely, this follows by applying interpolation on (21). Write $g_{n}(\theta)=$ $f_{n}(\theta)-e^{i \theta}$. By relabeling, if needed, we may assume that for all $n \geq 1$ 
we have $\left\|g_{n}\right\|_{C^{1, \alpha}(\mathbb{T})} \leq 1 / 10$, say. Since Id $: \mathbb{T} \rightarrow \mathbb{C}$ is 1 -bi-Lipschitz, and the Lipschitz norm of $g_{n}$ is small we obtain that $f_{n}: \mathbb{T} \rightarrow \partial \Omega_{n}$ is a diffeomorphism. We simply define $\Psi_{n}$ as the harmonic extension

$$
\begin{aligned}
\Psi_{n}(z) & =\frac{1}{2 \pi} \int_{0}^{2 \pi} P\left(z, e^{i t}\right) f_{n}\left(e^{i t}\right) d t=z+\frac{1}{2 \pi} \int_{0}^{2 \pi} P\left(z, e^{i t}\right) g_{n}\left(e^{i t}\right) d t \\
& =z+G_{n}(z), \quad z \in \mathbb{D} .
\end{aligned}
$$

Since $\left\|g_{n}^{\prime}\right\|_{\infty} \rightarrow 0$ and $\left\|H g_{n}^{\prime}\right\|_{\infty} \rightarrow 0$ (recall that the Hilbert transform $H$ is continuous in $C^{\alpha}(\mathbb{T})$ ), we may also assume that $\left|D G_{n}(z)\right| \leq 1 / 2$ for all $n$, and we have $\lim _{n \rightarrow \infty}\left\|D G_{n}\right\|_{L^{\infty}(D)}=0$. Especially, $\Psi_{n}: \overline{\mathbb{D}} \rightarrow \overline{\Omega_{n}}$ is $C^{1}$ and bi-Lipschitz, hence diffeomorphism. The first condition in (13) follows immediately, and condition (14) is immediate since $\Psi_{n}$ is harmonic. It remains to verify the second condition in (13). For that end observe that by [21] the fact that $\left\|g_{n}\right\|_{C^{1, \alpha}(\mathbf{T})} \leq C$ for all $n$ implies that the Poisson extension satisfies

$$
\left\|D^{2} G_{n}(z)\right\| \leq \frac{C^{\prime}}{(1-|z|)^{1-\alpha}} .
$$

This obviously yields the desired uniform bound for $\left\|D^{2} G_{n}\right\|_{L^{p}(\mathbb{D})}$ if we take $p<(1-\alpha)^{-1}$.

Another condition is obtained by specializing to Riemann maps - the proof of the the preceding theorem could also be based on certain results of Smirnov concerning the regularity of conformal extensions and the following lemma:

Lemma 4. Let $p>2$. The sequence of bounded Jordan domains $\Omega_{n} \subset \mathbb{C}$ converges in $W^{1, p}$-controlled sense to the unit disc $\mathbb{D}$ if the Riemann maps $F_{n}: \mathbb{D} \rightarrow \Omega_{n}$ (normalized by $F_{n}(0)=0$ and $\arg F_{n}^{\prime}(0)>0$ ) satisfy

$$
\lim _{n \rightarrow \infty}\left\|F_{n}^{\prime}-1\right\|_{L^{\infty}(\mathbb{D})}=0, \quad \text { and } \quad\left\|F_{n}^{\prime \prime}\right\|_{L^{p}(D)} \leq M_{0} \text { for all } n \geq 1,
$$

with some $M_{0}<\infty$.

Proof. Obvious after the definition of controlled convergence.

Remark 3.1. It is an open question whether one can weaken the condition $\alpha>1 / 2$ in Theorem 2 .

\section{Proof of Theorem 3 and Corollary 1: A SMirnov theorem for QC-MAPS}

Proof of Theorem 3. We first assume that $f$ is as in the theorem so that $\Delta f(z) \leq(1-|z|)^{-a}$ with $a \in(0,1)$. Then we are to show that the boundary map induced by $w$ is absolutely continuous. For that end we need two simple lemmas. 
Lemma 5. Assume that $u \in C(\overline{D)}$ is a harmonic mapping of the unit disk into $\mathbf{C}$ such that the the $U:=u_{\mid \mathbf{T}}$ is a homeomorphism and $U(\mathbf{T})=\Gamma$ is a rectifiable Jordan curve. Then $\left|\Gamma_{r}\right|:=\int_{\mathrm{T}}\left|\partial_{\theta} u\left(r e^{i \theta}\right)\right| d \theta$ is increasing in $r$ so that $\left|\Gamma_{r}\right| \leq|\Gamma|$. Especially, the angular derivative of u satisfies $\partial_{\theta} u(z) \in h^{1}$.

Proof of Lemma 5 By differentiating the Fourier-series representation

$$
u\left(r e^{i \theta}\right)=\sum_{n=-\infty}^{\infty} \widehat{g}_{n} r^{|n|} e^{i n \theta}
$$

we see immediately that $\partial_{\theta} u(z)$ is the harmonic extension to $U$ of the distributional derivative $\partial_{\theta} g$. By assumption, $g$ is of bounded variation, and hence $\partial_{\theta} g$ is a finite (signed) Radon measure, which implies that $\partial_{\theta} u \in h^{1}$. It is well-known (see [20, 11.17]) that for functions in $h^{1}$ the integral average $\int_{\mathbf{T}}\left|\partial_{\theta} u\left(r e^{i \theta}\right)\right| d \theta$ is increasing in $r$.

Lemma 6. Let $g \in L^{p}(\mathbf{U})$ with $p>1$. Then there is a unique solution to the Poisson equation $\Delta v=g$ such that $v \in C(\bar{U})$ and $v_{\mid \mathbf{T}}=0$. Moreover, the weak derivative $D v$ can be modified in a set of measure zero so that

$$
\int_{0}^{2 \pi}\left|D u\left(r e^{i \theta}\right)\right| d \theta \leq C(g)<\infty \quad \text { for } r \in(1 / 2,1) .
$$

Proof. The classical regularity theory for elliptic equations (see [1],[8]) yields a quick approach, as it guarantees that our Poisson equation has a unique solution $v$ in the Sobolev space $W^{2, p}(U)$ (which is of course given by the Green potential, see (2)) and we have continuity up to the boundary. The derivatives satisfy $\partial_{z}, \partial_{\bar{z}} \in W^{1, p}(U)$. Especially, we then have $\|D v\|_{W^{1, p}}(B(0, r)) \leq C^{\prime}$ for any $r \in(1 / 2,1)$. At this stage the trace theorem (see e.g. [23]) for the space $W^{1, p}(U)$ and a simple scaling argument shows for a suitable representative of $D v$ that

$$
\left\|(D v)_{r}\right\|_{B_{p, p}^{1-1 / p}(\mathbb{D})} \leq C^{\prime} \quad \text { for } r \in(1 / 2,1) .
$$

Here $(D v)_{r}$ stands for the function $\mathbf{T} \ni \theta \mapsto v\left(r e^{i \theta}\right)$. The claim follows by observing the continous imbeddings $B_{p, p}^{1-1 / p}(\mathbb{D}) \subset L^{p}(\mathbb{D}) \subset L^{1}(\mathbb{D})$.

Recall also that any analytic (or anti-analytic) function in $h^{1}$ can be represented as the Poisson integral of an $L^{1}$-function, see [20, Theorem 17.11] or [9]. In order to proceed towards the absolute continuity of boundary values of $f$, write $f=a+\bar{b}+v$, where $v$ solves $\Delta v=g:=\Delta f$ with $v_{\mid \mathbf{T}}=0$ and $a$ and $b$ are analytic in the unit disk. Since $u:=a+\bar{b}=\mathcal{P}\left[f_{\mid \mathbf{T}}\right]$, where $f_{\mid \mathbf{T}}$ is a homeomorphism, it follows from Lemma 5 that $\partial_{\theta} u=i\left(z a^{\prime}-\overline{z b^{\prime}}\right) \in h^{1}(\mathbf{U})$, because $f(\mathbf{T})$ is a rectifiable curve. Further, the weak derivatives satisfy

$$
f_{z}=a^{\prime}+v_{z}, \quad f_{\bar{z}}=\overline{b^{\prime}}+v_{\bar{z}}
$$


Now we use that

which implies that

$$
\left|f_{\bar{z}}\right| \leq k\left|f_{z}\right|, \quad k=\frac{K-1}{K+1}
$$

$$
\left|a^{\prime}+v_{z}\right| \leq k\left|b^{\prime}+\overline{v_{\bar{z}}}\right|
$$

As

$$
b^{\prime}=\frac{\bar{z}}{z} \overline{a^{\prime}}-\frac{i}{z} \overline{u_{\theta}}
$$

we obtain for $z \neq 0$ that

$$
\left|a^{\prime}\right| \leq k\left|\frac{\bar{z}}{z} \bar{a}^{\prime}-\frac{i}{z} \overline{u_{\theta}}+\overline{v_{\bar{z}}}\right|+\left|v_{z}\right| .
$$

This yields for $|z| \geq 1 / 2$ the inequality, valid almost everywhere

$$
\left|a^{\prime}\right| \leq \frac{1}{1-k}\left(2\left|\overline{u_{\theta}}\right|+\left|\overline{v_{\bar{z}}}\right|+\left|v_{z}\right|\right) \text {. }
$$

Our assumption on the size of the Laplacian of $f$ yields that $\Delta f \in L^{p}(\mathbb{D})$ for some $p>1$. By combining this with above inequality, and noting that $\overline{u_{\theta}} \in h^{1}$ by Lemma 5, we infer (using simple argument that uses Fubini as the above inequality holds only for a.e. $z$ ) that $a^{\prime} \in H^{1}$. Then the relation $b^{\prime}=\frac{\bar{z}}{z} \overline{a^{\prime}}-\frac{i}{z} \overline{u_{\theta}}$ verifies that also $b \in H^{1}$. Thus $\partial_{\theta} u$ is the Poisson integral of an $L^{1}$ function, and we conclude that $f_{\mid \mathbf{T}}=u_{\mid \mathbf{T}}$ is absolutely continuous.

In order to prove the optimality of Theorem 3, we are to construct quasiconformal maps with non-absolutely continuous boundary values, but at the same time with not too large Laplacian. For that end it is easier to work in the upper half space $\mathbb{C}^{+}:=\{z: \operatorname{Im} z>0\}$. We will produce the desired functions with the help of Zygmund measures. Recall first that a bounded and continuous function $g: \mathbb{R} \rightarrow \mathbb{R}$ is Zygmund if

$$
|g(x+t)+g(x-t)-2 g(x)| \leq C|t| \text { for all } x, t \in \mathbb{R} .
$$

The smallest possible $C$ above is the Zygmund norm of $g$. If $g$ is increasing, its derivative is a positive finite Borel measure, $g^{\prime}=\mu$, on $\mathbb{R}$ and we call $g$ a singular Zygmund function if, in addition, $\mu$ is singular. It is well-known that there exists singular Zygmund measures, see [19] or [10]. In general, we refer the reader to the interesting article [3] for further information on this type of measures.

We next recall a modified version of the Beurling-Ahlfors extension, due to Fefferman, Kenig and Pipher [7]. For that end denote the Gaussian density by $\psi(x):=(2 \pi)^{-1 / 2} e^{-x^{2} / 2}$, and notice that $-\psi^{\prime}(x)=-x \psi(x)$. As usual, for $t>0$ we define the dilation $\psi_{t}(x):=t^{-1} \psi(x / t)$, and $\psi_{t}^{\prime}$ is defined in analogous way. Then the extension $u$ of and (at most polynomially) increasing homeomorphism $g: \mathbb{R} \rightarrow \mathbb{R}$ is defined by setting

$$
u(x+i t):=\left(\psi_{t} * g\right)(x)+i\left(-\psi_{t}^{\prime} * g\right)(x), \quad \text { for all } \quad x+i t \in \mathbb{C}^{+} .
$$


Obviously, $u$ is smooth in $\mathbb{C}^{+}$and it has the right boundary values. We have:

Lemma 7 ([7], Lemma 4.4.]). If $g: \mathbb{R} \rightarrow \mathbb{R}$ is quasisymmetric, then the extension u defined via (23) defines a quasiconformal homeomorphism of $\mathbb{C}^{+}$whose boundary map coincides with $g$.

We need one more auxiliary result:

Lemma 8. Assume that $g: \mathbb{R} \rightarrow \mathbb{R}$ is Zygmund. Then the extension (23) of $g$ satisfies for alla $x \in \mathbb{R}$ and $t>0$

$$
\begin{aligned}
& |\Delta u(x+i t)| \leq C t^{-1}, \quad \text { and } \\
& |\nabla u(x+i t)| \leq C \max \left(1, \log \left(t^{-1}\right)\right),
\end{aligned}
$$

where $C>0$ is a constant.

Proof. Let us first observe that if $g$ is Zygmund, then for any $\varphi \in W^{2,1}(\mathbb{R})$ (i.e. $\varphi, \varphi^{\prime \prime} \in L^{1}(\mathbb{R})$ ) we have

$$
\left\|\frac{d^{2}}{d x^{2}} \varphi_{t} * g\right\|_{L^{\infty}(\mathbb{R})}=O\left(t^{-1}\right), \quad \text { for all } t>0 .
$$

We note that this follows easily from the mere definition of Zygmund functions if $\varphi$ is even, but for general $\varphi$ we shall use the fact that $g$ can be decomposed as the sum $g=\sum_{j=0}^{\infty} g_{j}$, where $\left\|g_{j}\right\|_{L^{\infty}(\mathbb{R})}=O\left(2^{-j}\right)$ and $\left\|g_{j}^{\prime \prime}\right\|_{L^{\infty}(\mathbb{R})}=$ $O\left(2^{j}\right)$ for all $j \geq 0$, see [21, Corollary 1, p. 256]. We may compute in two ways

$$
\frac{d^{2}}{d x^{2}}\left(\varphi_{t} * g(x)\right)=\int_{-\infty}^{\infty} \varphi_{t}(x-y) g^{\prime \prime}(y) d y=t^{-2} \int_{-\infty}^{\infty} \varphi_{t}^{\prime \prime}(x-y) g(y) d y .
$$

By assuming first that $t \leq 1$ with $t \sim 2^{-k}$ we apply the first formula above to the sum $g=\sum_{j=0}^{k} g_{j}$, and the second one to the remainder $g=\sum_{j=k+1}^{\infty} g_{j}$. By noting that $\int_{-\infty}^{\infty}\left|\varphi_{t}(y)\right| d y=O(1)$ and $\int_{-\infty}^{\infty}\left|\varphi_{t}^{\prime \prime}(y)\right| d y=O(1)$, we obtain

$$
\left|\frac{d^{2}}{d x^{2}}\left(\varphi_{t} * g(x)\right)\right|=O\left(\sum_{j=1}^{k} 2^{j}+t^{-2} \sum_{j=k+1}^{\infty} 2^{-j}\right)=O\left(t^{-1}\right),
$$

which proves (24) for $t \in(0,1]$. If $t>1$ we simply apply the second formula directly on the bound $\|g\|_{L^{\infty}(\mathbb{R})}<\infty$ and obtain $\left\|\frac{d^{2}}{d x^{2}}\left(\varphi_{t} * g\right)\right\|_{L^{\infty}(\mathbb{R})} \leq O\left(t^{-2}\right)=$ $O\left(t^{-1}\right)$ for $t>1$.

We then consider the Laplacian of the extension $u$ of $g$. Since $\psi, \psi^{\prime} \in$ $W^{2,1}(\mathbb{R})$, we obtain immediately from (24) that $\left|\frac{d^{2}}{d x^{2}} u(x+i t)\right|=O\left(t^{-1}\right)$ uniformly in $x \in \mathbb{R}$. In turn, to consider differentiation with respect to $t$, assume 
that $\phi: \mathbb{R} \rightarrow \mathbb{R}$ is smooth and $\left(1+|t|^{2}\right) \phi(t)$ is integrable. Then

$$
\begin{aligned}
\frac{d}{d t} \varphi_{t} * g(x) & =\int_{-\infty}^{\infty}\left(-t^{-2} \varphi_{t}(x-y)-t^{-3}(x-y) \varphi_{t}^{\prime}(x-y)\right) g(y) d y \\
& \left.=\int_{-\infty}^{\infty} g(y) \frac{d}{d y}\left(t^{-2}(x-y) \varphi_{t}(x-y)\right)\right) d y \\
& =-t^{-1} \int_{-\infty}^{\infty} \frac{(x-y)}{t} \varphi\left(\frac{x-y}{t}\right) g^{\prime}(y) d y \\
& =\left(\varphi_{1}\right)_{t} * g^{\prime}(x),
\end{aligned}
$$

where $\varphi_{1}(y):=-y \varphi(y)$. An iteration gives, by denoting $\varphi_{2}(y):=y^{2} \varphi(y)$,

$$
\frac{d^{2}}{d t^{2}}\left(\varphi_{t} * g(x)\right)=\left(\varphi_{2}\right)_{t} * g^{\prime \prime}(x)=\frac{d^{2}}{d x^{2}}\left(\left(\varphi_{2}\right)_{t} * g(x)\right) .
$$

Since all the functions $t \psi(t), t^{2} \psi(t), t \psi(t), t^{2} \psi(t)$ and their second derivatives are integral, we may apply (25) and obtain as before the desired estimate for $\frac{d^{2}}{d t^{2}} u(x+i t)$.

The stated estimate for $\nabla u$ is proven in a similar way. We use the fact that for in the decomposition $g=\sum_{j=0}^{\infty} g_{j}$, one may in addition demand that $\left\|g_{j}^{\prime}\right\|_{\infty} \leq C$ for all $j \geq 1$ (see [22, Formula (53), p. 254]), which yields as before for $t \sim 2^{-k}<1$

$$
\left|\frac{d}{d x}\left(\varphi_{t} * g(x)\right)\right|=O\left(\sum_{j=1}^{k} 1+t^{-1} \cdot \sum_{j=k+1}^{\infty} 2^{-j}\right)=O\left(\log \left(t^{-1}\right)\right) .
$$

The case $t \geq 1$ is trivial, and the case of the $t$-derivative is reduced to estimating the $x$-derivative as before.

After these preparations it is now a simple matter to produce the desired example. Let $g_{0}$ be a singular Zygmund function which is constant outside $[-1,1]$ so that Set $g(x)=x+g_{0}(x)$ for $x \in \mathbb{R}$. As $g_{0}$ is Zygmund, the function $g$ is quasi symmetric. Then its Fefferman-Kenig-Pipher extension $u: \mathbb{C}^{+} \rightarrow \mathbb{C}^{+}$is quasiconformal with non-absolutely continuous boundary values over $[-1,1]$. Since the extension of the linear function $x \mapsto x$ is linear, we see that the Laplacian of $u$ equal that of the extension of $g_{0}$, and by the previous lemma we obtain the estimate

$$
|\Delta u(x+i t)| \leq C t^{-1} \quad \text { for all } x+i t \in \mathbb{C}^{+} .
$$

Next, let $h: \mathbb{D} \rightarrow \Omega^{\prime}$ be conformal, where $\Omega^{\prime}$ is a bounded and smooth Jordan domain that is contained in the upper half space $\mathbb{C}^{+}$and contains $[-2,2]$ as a boundary segment. Denote $\Omega=u\left(\Omega^{\prime}\right)$ so that $\Omega$ is smooth by our construction. Finally, pick a conformal map $\widetilde{h}: \Omega \rightarrow \mathbb{D}$ and define $f:=u \circ h$. Function $f$ satisfies all the requirements since the main terms in the formula for the Laplacian of $f$ (compare to (20) ) are $|\Delta u|$ and $|\nabla u|^{2}$, 
and the previous lemma also yields suitable bounds also for the gradient term.

Proof of Corollary 1 The example for optimality constructed above obviously works also for the Corollary. In a similar vain, the proof of the positive direction of Theorem 3 also applies as such for the Corollary since in the proof we used as a starting point the fact that $\Delta u \in L^{p}(\mathbb{D})$ for some $p>1$.

Remark 4.1. There exists singular Zygmund functions on the real line such that $g(x+t)+g(x-t)-2 g(x)=o(t)$ with quantitative little $o$ in the right hand side - the derivatives of such functions are sometimes called Kahane measures. A possible decay of the right hand side is $o\left(t \log ^{-1 / 2}(1 / t)\right)$ for small $t$, but one cannot decrease the power of $\log$ here. Using this kind of measures in our construction gives examples with Laplacian growth $o\left(t^{-1}\right)$, where the little $o$ can be made explicit.

However, it is an open problem whether Corollary 1 is true for the exponent $p=1$, as merely implementing the Kahane measures described above appears not to give enough extra decay for the Laplacian.

\section{REFERENCES}

[1] S. Agmon, A. Douglis, and L. Nirenberg: Estimates near the boundary for Elliptic Partial Differential Equations Satisfying General Bounday Conditions. I, Comm. Pure and Appl. Math. 12 (1959) 623-727.

[2] L. Ahlfors: Lectures on Quasiconformal mappings, Van Nostrand Mathematical Studies, D. Van Nostrand 1966.

[3] A.B. Aleksandrov, J.M. Anderson and A Nicolau: Inner functions, Bloch spaces and symmetric measures, Proc. London Math. Soc. 79 (1999), 318-352.

[4] K. Astala, T. Iwaniec and G. J. Martin: Elliptic partial differential equations and quasiconformal mappings in the plane, Princeton University Press, 2009.

[5] K. Astala and V. Manojlović: On Pavlović theorem in space, ArXiV Math 1410.7575

[6] K. Astala, T. Iwaniec, I. Prause and E. Saksman: Bilipschitz and quasiconformal rotation, stretching and multifractal spectra, to appear in Publ. Math. Inst. Hautes Études Sci.

[7] R.A. Fefferman, C.E. Kenig and J. Pipher: The theory of weights and the Dirichlet problem for elliptic equations, Ann. of Math. 134 (1991), 65-124.

[8] D. Gilbarg and N. Trudinger: Elliptic Partial Differential Equations of Second Order. 2 Edition, Springer 1977, 1983.

[9] G. L. Goluzin: Geometric function theory. Nauka, Moskva 1966.

[10] J. P. Kahane: Trois notes sur les ensembles parfait linearés, Enseign. Math. 15 (1969) 185-192.

[11] D. Kalaj, M. Markovic and M. Mateljević: Carathéodory and Smirnov type theorems for harmonic mappings of the unit disk onto surfaces, Ann. Acad. Sci. Fenn. Math. 38 (2013), 565-580.

[12] D. Kalaj: Harmonic mappings and distance function. Ann. Sc. Norm. Super. Pisa, Cl. Sci. (5) 10 (2011), 669-681. 
[13] D. Kalaj: On boundary correspondences under quasiconformal harmonic mappings between smooth Jordan domains. Math. Nachr. 285, No. 2-3, 283-294 (2012).

[14] D. Kalaj: A priori estimate of gradient of a solution to certain differential inequality and quasiregular mappings, Journal d'Analyse Mathematique 119 (2013), 63-88.

[15] D. Kalaj, M. Pavlović: On quasiconformal self-mappings of the unit disk satisfying the Poisson equation, Trans. Amer. Math. Soc. 363 (2011) 4043-4061.

[16] C. Pommerenke: Boundary behaviour of conformal maps. Grundlehren der Mathematischen Wissenschaften. 299. Berlin: Springer- Verlag. ix, 300 p. (1992).

[17] D. Partyka and K. Sakan: On bi-Lipschitz type inequalities for quasiconformal harmonic mappings, Ann. Acad. Sci. Fenn. Math.. Vol 32, pp. 579-594 (2007).

[18] M. Pavlović: Boundary correspondence under harmonic quasiconformal homeomorfisms of the unit disc, Ann. Acad. Sci. Fenn., Vol 27, (2002) 365-372.

[19] G. Piranian: Two monotonic, singular, uniformly almost smooth functions, Duke Math. J. 33 (1966), 255-262.

[20] W. Rudin: Real and complex analysis. Third edition. McGraw-Hill 1986.

[21] E. M. Stein: Singular integrals and differentiability properties of functions. Princeton Mathematical Series, No. 30 Princeton University Press, Princeton, N.J. 1970

[22] E. M. Stein: Harmonic analysis: real-variable methods, orthogonality, and oscillatory integrals. With the assistance of Timothy S. Murphy. Princeton Mathematical Series, 43. Princeton University Press, Princeton, NJ, 1993.

[23] H. Triebel: Interpolation theory, function spaces, differential operators. 2. Auflage. Barth, Heidelberg 1995.

University of Montenegro, Faculty of Natural Sciences and Mathematics, Cetinjski PUT B.B. 81000 Podgorica, MonteNEGro

E-mail address: davidkalaj@gmail.com

Department of Mathematics and Statistics, University of Helsinki, PO Box 68, FI00014 Helsinki, Finland

E-mail address: eero.saksman@helsinki.fi 